\title{
Coeficiente de cultura e produtividade da cebola submetida a diferentes lâminas de irrigação
}

\author{
Gertrudes M. de Oliveira', Mário M. V. B. R. Leitão², Regiane de C. Bispo', \\ Irai M. S. Santos' ${ }^{1}$ Caio B. de A. Lima' \& Adheilton R. P. de Carvalho ${ }^{1}$
}

\begin{abstract}
RESUMO
O presente trabalho teve como objetivo determinar coeficientes de cultura e avaliar a produtividade da cebola submetida a diferentes lâminas de irrigação. O experimento foi conduzido no campo experimental do DTCS/ UNEB, em Juazeiro, no período de abril a setembro de 2011. Para determinação da ETc foram efetuadas medidas diárias em evapotranspirômetros. A ETo foi obtida através dos métodos do tanque classe A (TCA) e Penman-Monteith (PM) e o Kc pela relação: Kc = ETc/ETo. O delineamento utilizado foi inteiramente casualizado, com três tratamentos e sete repetições. As variáveis analisadas foram: diâmetro transversal, peso médio de bulbos, produção total, produção comercial, teor de sólidos solúveis totais e acidez total titulável. Os Kc's encontrados tomando-se como base a ETo determinada pelo método TCA, com o Kp calculado com dados climáticos obtidos in situ, foram bem próximos daqueles determinados pelo método PM; a produtividade total superou a média nacional em torno de $122 \%$ para os tratamentos $\mathrm{T}_{1}$ (irrigação feita na base de $\mathrm{ET}_{\mathrm{c}}$ ) $\mathrm{e}_{3}$ (irrigação em base na $\mathrm{ET}_{0}$ calculado pelo método de $\mathrm{PM}$ ) e em $86 \%$ para o tratamento $\mathrm{T}_{2}$ (irrigação na base de $\mathrm{ET}_{\mathrm{o}}$ calculado pelo método de TCA); o manejo de irrigação, tomando-se como base o método TCA, pode proporcionar produtividade elevada levando-se em consideração as condições climáticas locais.
\end{abstract}

Palavras-chave: evapotranspiração da cultura, evapotranspiração de referência, condições climáticas

\section{Productivity and crop coefficient of onion under different irrigation levels}

\begin{abstract}
The present study aimed to determine crop coefficients and evaluate the productivity of onion under different irrigation levels. The experiment was conducted in the experimental field at DTCS/UNEB Juazeiro during the period from April to September 2011. For determining the ETc, daily measurements were made in evapotranspirometers. The ETo was obtained by the methods of the Class A pan (TCA) and PenmanMonteith (PM) and the Kc by: $\mathrm{Kc}=\mathrm{ETc} / \mathrm{ETo}$. The experimental design was completely randomized with three treatments and seven replications. The examined variables were: transverse diameter, mean bulb weight, total production, commercial production, total soluble solids and titratable acidity. The Kc values found by taking as a basis the ETo determined by the TCA method with the Kp calculated using weather data obtained in situ, were very close to those determined by the PM method; the total productivity surpassed the national average by $122 \%$ for $\mathrm{T}_{1}$ (irrigation on the basis of $\mathrm{ET}_{\mathrm{c}}$ ) and $\mathrm{T}_{3}$ (irrigation on the basis of $\mathrm{ET}_{0}$ calculated according to PM) treatments and $86 \%$ for the $\mathrm{T}_{2}$ (irrigation on the basis of $\mathrm{ET}_{0}$ calculated according to TCA) treatment; the irrigation management, based on the TCA method can provide high productivity, taking into account local climatic conditions.
\end{abstract}

Key words: crop evapotranspiration, reference evapotranspiration, climatic conditions

'Departamento de Tecnologia e Ciências Sociais/UEBA, Av. Edgard Chastinet, s/n, Bairro São Geraldo, CEP 48900-000, Juazeiro, BA, Fone: (74) 3611-7362. E-mail: gemoliveira@uneb.br; regiane.carvalho_bispo@hotmail.com; irai.manuela@gmail.com; caio_004@hotmail.com, adheilton_92@hotmail.com

${ }^{2}$ Colegiado de Engenharia Agrícola e Ambiental/ Universidade Federal do Vale do São Francisco, Av. Antônio Carlos Magalhães, 510, Santo Antônio, CEP 48902-300, Juazeiro, BA, Fone: (74) 2102 7604. E-mail: mario.miranda@univasf.edu.br 


\section{INTRODUÇÃO}

A cebola (Allium cepa L.) é a hortaliça mais difundida no mundo e se destaca entre as demais cultivadas, tanto pelo seu volume de produção como pelo consumo e valor econômico. A cultura ocupa o terceiro lugar em importância econômica no mundo e o Brasil é o $8^{\circ}$ maior produtor participando com cerca de $2 \%$ da oferta mundial (Coopercitrus, 2012). Em 2011 a produtividade média nacional, de acordo com o IBGE (2012), se manteve em torno de $23.278 \mathrm{~kg} \mathrm{ha}^{-1}$. A região Nordeste, devido às condições edafoclimáticas, oferece grandes vantagens comparativas às demais regiões produtoras de cebola do país, uma vez que permite o plantio durante o ano todo (Grangeiro et al., 2008).

Um dos fatores mais importantes para o desenvolvimento de qualquer espécie é a água, cuja falta caracteriza uma das principais restrições ao crescimento e desenvolvimento das espécies cultivadas (Lopes et al., 2011). Em se tratando da cebola, de acordo com Marouelli et al. (2005), com exceção da região Sul, o cultivo é totalmente realizado com o uso da irrigação, prática fundamental para melhor qualidade da bulbificação. De acordo com Vilas-Boas et al. (2011), o manejo correto da irrigação se torna indispensável uma vez que pode ser ajustado às condições momentâneas da cultura. Muitos autores reportaram que a produtividade de bulbos da cebola é altamente dependente da quantidade de água aplicada (SantaOlalla et al., 1994; Saha et al., 1997; Shock et al., 2000). Apesar desta dependência, são poucas as informações acerca das reais necessidades hídricas da cultura, que possam subsidiar o manejo das irrigações e, desta forma, promover maiores rendimentos.

O consumo de água de uma cultura depende diretamente da demanda energética atmosférica, do conteúdo de água no solo e da resistência da planta à perda de água para a atmosfera. No que se refere ao manejo da irrigação, a base para a quantificação da água a ser aplicada a determinada cultura está associada, comumente, à capacidade da superfície do solo e da vegetação de perder água para a atmosfera (Silva et al., 2011). Ou seja, considerar os processos de evaporação do solo e de transpiração das plantas, conjuntamente denominados evapotranspiração. A evapotranspiração da cultura (ETc) é uma variável básica da irrigação e depende dos elementos meteorológicos, da cultura e do solo podendo ser medida diretamente (lisímetros) ou indiretamente (equações combinadas) por vários métodos (Chaves et al., 2005).

Uma forma usual de se estimar a ETc é a partir da evapotranspiração de referência (ETo), por meio de coeficientes apropriados conhecidos como coeficiente de cultura $(\mathrm{Kc})$. Segundo Allen et al. (1998), o Kc representa uma integração dos efeitos de quatro características primárias que distinguem uma cultura específica da de referência, tais como: altura, albedo, propriedades aerodinâmicas e da folha e evaporação do solo. Diversos têm sido os estudos voltados para a determinação desses coeficientes; entretanto, Doorenbos \& Pruitt (1977) enfatizam a necessidade de calibração dos coeficientes de cultura sob condições climáticas específicas e Allen et al. (1998) recomendam que os valores de Kc e da duração dos estádios da cultura sejam ajustados por meio de experimentos para cada região, de acordo com a variedade plantada, condições climáticas e as técnicas de cultivo utilizadas.
Apesar da existência de diversos métodos de estimativas da ETo, muitas vezes estes são utilizados em condições climáticas e agronômicas diferentes daquelas em que foram inicialmente concebidos sendo, portanto, de extrema importância avaliar o grau de exatidão desses modelos antes de utilizá-los para nova condição (Sousa et al., 2010).

Entre os diferentes métodos propostos na literatura para determinação da ETo, por sua praticidade, o método do tanque Classe A recomendado pela FAO é utilizado em projetos de irrigação. Entretanto, por considerar um número maior de variáveis climatológicas, a FAO recomenda o método de Penman-Monteith como padrão para a estimativa de ETo (Allen et al., 1998).

Tendo em vista que para o manejo eficiente da irrigação a quantidade de água aplicada deve ser compatível com as necessidades de consumo da cultura e considerando, ainda, que estudos referentes à produção de cebola irrigada por gotejamento são escassos (Vilas Boas et al., 2012), o presente trabalho teve como objetivo determinar os coeficientes de cultura e avaliar a produtividade da cebola submetida a diferentes lâminas de irrigação no município de Juazeiro, BA.

\section{Material e Métodos}

O experimento foi realizado no período de abril a setembro de 2011 no campo experimental do Departamento de Tecnologia e Ciências Sociais - DTCS da Universidade do Estado da Bahia - UNEB, em Juazeiro (Lat. 09² 24' 50”' S; Long. 40³0' 10" W; Alt. $368 \mathrm{~m})$. Uma estrutura de evapotranspirômetros de lençol freático constante $\left(5,0 \mathrm{~m}^{2}\right.$ e $1,30 \mathrm{~m}$ de profundidade $)$ foi construída no centro de uma área experimental de $1350 \mathrm{~m}^{2}$ preparada em sistema de plantio em sulcos espaçados $80 \mathrm{~cm}$ e parcelas com $0,40 \times 20,0 \mathrm{~m}$. O solo da área experimental, de acordo com o Laboratório de Análises de Solo, Água e Calcário - LASAC do DTCS/UNEB foi classificado como Neossolo Flúvico. O sistema de irrigação utilizado foi gotejamento com o espaçamento de $30 \mathrm{~cm}$ entre gotejadores.

Para determinação da evapotranspiração da cultura foram efetuadas medidas diárias nos evapotranspirômetros. A evapotranspiração de referência (ETo) foi determinada com base em dados obtidos na estação meteorológica automática instalada em frente à área experimental e dados de evaporação do tanque Classe A (ECA) - ETo (TCA), através da relação:

$$
\mathrm{ETo}=\mathrm{Kp} \mathrm{ECA}
$$

em que:

Kp - é o coeficiente de tanque obtido pela expressão proposta por Snyder (1992):

$$
\mathrm{Kp}=0,482+0,024 \operatorname{Ln}(\mathrm{B})-0,000376 \mathrm{U}+0,0045 \mathrm{UR}
$$

em que:

$\mathrm{U} \quad$ - é a velocidade do vento em $\mathrm{km} \mathrm{d}^{-1}$

UR - a umidade relativa do ar média diária em \%

B - o tamanho da bordadura em $\mathrm{m}$ 
A ETo foi determinada também pelo método de PenmanMonteith parametrizado pela FAO (Allen et al., 1998) - ETo (PM).

$$
\text { ETo }=\frac{0,408 \Delta(\mathrm{Rn}-\mathrm{G})+\frac{\gamma 900 \mathrm{U}_{2}(\mathrm{es}-\mathrm{ea})}{\mathrm{T}+273}}{\Delta+\gamma\left(1+0,34 \mathrm{U}_{2}\right)}
$$

em que:

$\mathrm{Rn}$ - radiação líquida total diária $\left(\mathrm{MJ} \mathrm{m}^{-2} \mathrm{~d}^{-1}\right)$

$\mathrm{G} \quad$ - fluxo de calor no solo $\left(\mathrm{MJ} \mathrm{m}^{-2} \mathrm{~d}^{-1}\right)$

$\gamma \quad$ - constante psicrométrica $\left(\mathrm{kPa}^{\circ} \mathrm{C}^{-1}\right)$

$\mathrm{U}_{2}$ - velocidade do vento a $2 \mathrm{~m}$ de altura $\left(\mathrm{m} \mathrm{s}^{-1}\right)$

es - pressão de saturação de vapor $(\mathrm{kPa})$

ea - pressão parcial de vapor $(\mathrm{kPa})$

$\mathrm{T}$ - temperatura média do ar $\left({ }^{\circ} \mathrm{C}\right)$

$\Delta \quad$ - declividade da curva de pressão de vapor em relação à temperatura do $\operatorname{ar}\left(\mathrm{kPa}^{\circ} \mathrm{C}^{-1}\right)$

O coeficiente de cultura $(\mathrm{Kc})$ foi determinado pela razão entre a evapotranspiração da cultura (ETc) e a de referência:

$$
\mathrm{Kc}=\frac{\mathrm{ETc}}{\mathrm{ETo}}
$$

A cultivar utilizada foi a Alfa São Francisco, desenvolvida pela Embrapa. A semeadura foi realizada em canteiros no dia 19/04/2011, e o transplantio na área experimental e dentro dos evapotranspirômetros 35 dias depois utilizando-se espaçamento de $0,10 \times 0,10 \mathrm{~m}$, comportando quatro fileiras de plantas por parcela; sobre cada parcela da área experimental foi instalada uma linha de gotejadores. Para a área útil foram descartadas as duas primeiras plantas de cada fileira e as filas laterais. A adubação, desde a fundação à cobertura, foi feita de acordo com o recomendado para a cultura e com base na análise de solo. Os tratos culturais e o controle fitossanitário utilizado foram semelhantes àqueles comumente empregados na cultura da cebola.

O delineamento estatístico utilizado foi inteiramente casualizado, com três tratamentos e sete repetições. Os tratamentos foram: $T_{1}$ - irrigação feita com base nos dados de evapotranspiração da cultura (ETc) observados nos evapotranspirômetros; $\mathrm{T}_{2}$ - irrigação efetuada tomando-se como base os coeficientes de cultura (Kc) propostos por Marouelli et al. (2005) para a cultura da cebola e a ETo obtida pelo método

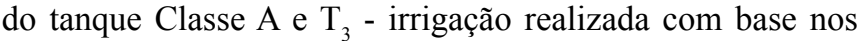
coeficientes propostos por Marouelli et al. (2005) e a ETo obtida pelo método de Penman-Monteith $(\mathrm{ETc}=\mathrm{Kc} \mathrm{ETo})$. A reposição de água no solo, conforme a lâmina evapotranspirada em cada tratamento foi efetuada diariamente através de irrigação por gotejamento.

O período de desenvolvimento da cultura foi dividido em quatro estádios: inicial (I) - do transplante das mudas até o estabelecimento inicial das plantas $(10 \%$ do crescimento vegetativo); vegetativo (II) - do estabelecimento inicial das plantas até o início da bulbificação; bulbificação (III) - início da bulbificação até o início da maturação; maturação (IV) - início da maturação dos bulbos até a colheita. Os coeficientes de cultura determinados com base nos dados de ETo (PM) e ETo (TCA) foram comparados entre si e com aqueles propostos por Marouelli et al. (2005) para a cultura da cebola.

A colheita foi realizada no dia 24 de setembro de 2011 quando a maioria das plantas se encontrava tombada (estalo). As plantas colhidas foram submetidas ao processo de cura ficando três dias expostas ao sol e doze dias à sombra em ambiente coberto e ventilado. Após a toalete dos bulbos foram feitas a classificação e pesagem dos bulbos. Os bulbos foram classificados, de acordo com o maior diâmetro transversal, nas classes 1 (diâmetro $<35 \mathrm{~mm}$, bulbos considerados não comerciais), 2 (35 a $50 \mathrm{~mm}), 3(50$ a $70 \mathrm{~mm})$ e 4 (70 a 90 $\mathrm{mm}$ ) (Costa et al., 2000). Foram avaliadas as seguintes características: diâmetro, peso médio de bulbos, produção total, produção comercial, teor de sólidos solúveis totais e acidez total titulável. A análise estatística foi realizada por meio da análise de variância dos dados (teste F) e da comparação de médias de tratamentos entre si (teste de Tukey, 0,05 de probabilidade).

\section{Resultados e Discussão}

Em relação às condições climáticas observadas no período de condução do experimento verificou-se que, em média, a radiação solar global foi de $20,6 \mathrm{MJ} \mathrm{m}^{2} \mathrm{~d}^{-1}$; a umidade relativa do ar, 57,4\%; a velocidade do vento, $207,7 \mathrm{~km} \mathrm{~d}^{-1}$; o total de precipitação pluviométrica, $3,9 \mathrm{~mm}$ e, em termos de temperatura, a média, $24,6{ }^{\circ} \mathrm{C}$, a máxima, $30,9^{\circ} \mathrm{C}$ e a mínima, $19,1^{\circ} \mathrm{C}$. Esses valores de temperatura estão compreendidos na faixa ideal para o desenvolvimento da cebola. Segundo Rezende et al. (2007), altas temperaturas (acima de $32^{\circ} \mathrm{C}$ ) na fase inicial de desenvolvimento das plantas podem provocar bulbificação prematura indesejável; ao contrário, a exposição das plantas a períodos prolongados de temperaturas baixas $\left(<10^{\circ} \mathrm{C}\right)$, pode induzir o florescimento prematuro ("bolting"), que é altamente indesejável, quando se visa à produção comercial de bulbos e não de sementes. Temperaturas em torno de 15,5 a $21,1{ }^{\circ} \mathrm{C}$ promovem formação de melhores bulbos e maior produção.

Durante a realização do experimento as maiores médias diárias da evapotranspiração da cultura (ETc) foram registradas no estádio III atingindo valores de até $6,2 \mathrm{~mm} \mathrm{~d}^{-1}$; para todo o período a média foi de $4,0 \mathrm{~mm} \mathrm{~d}^{-1}$ e o total de $487,9 \mathrm{~mm}$. Em termos de total de água, o resultado está de acordo com Marouelli et al. (2005), que colocam que a necessidade total de água da cultura pode variar de 350 a $650 \mathrm{~mm}$ dependendo das condições climáticas e do ciclo da cultivar.

A evapotranspiração média da cultura (ETc), a evapotranspiração média de referência (ETo) determinada pelos métodos do tanque Classe A (TCA) e Penman-Monteith (PM) e os respectivos coeficientes de cultura $(\mathrm{Kc})$ para cada estádio de desenvolvimento, bem como os coeficientes de cultura propostos por Marouelli et al. (2005), para a cebola (Kc - M), são apresentados na Tabela 1. Verifica-se, nesta tabela que, em média, o maior consumo de água pela cultura ocorreu no estádio III, 5,1 mm, estádio que corresponde ao maior desenvolvimento vegetativo da cultura e à formação de bulbos.

Comparando os valores de ETo estimados pelos métodos do tanque Classe A e Penman-Monteith verifica-se, na Tabela 
Tabela 1. Evapotranspiração média da cultura (ETc), evapotranspiração média de referência determinada pelos métodos do tanque Classe A (ETo - TCA) e Penman-Monteith (ETo - PM) e coeficientes de cultura determinados com base na ETo - TCA (Kc -TCA) e ETo PM (Kc-PM) e propostos por Marouelli et al. (2005) (Kc - M) para os diferentes estádios de desenvolvimento da cebola

\begin{tabular}{ccccccc}
\hline \multirow{2}{*}{ Estádios } & ETc & $\begin{array}{c}\text { ETo } \\
\text { (TCA) }\end{array}$ & $\begin{array}{c}\text { ETo } \\
\text { (PM) }\end{array}$ & $\begin{array}{c}\text { Kc } \\
\text { (TCA) }\end{array}$ & $\begin{array}{c}\text { Kc } \\
\text { (PM) }\end{array}$ & $\begin{array}{c}\text { Kc } \\
\text { (M) }\end{array}$ \\
\cline { 2 - 4 } I & 2,4 & 4,6 & 4,5 & 0,53 & 0,55 & 0,60 \\
II & 3,0 & 4,8 & 4,7 & 0,62 & 0,64 & 0,80 \\
III & 5,1 & 5,3 & 5,4 & 0,96 & 0,96 & 0,95 \\
IV & 4,1 & 6,1 & 6,3 & 0,68 & 0,66 & 0,65 \\
\hline
\end{tabular}

1, que os valores se aproximaram para todos os estádios, com pequena tendência de superestimativa dos valores de ETo (TCA) nos estádios I e II e subestimativa nos estádios III e IV. De acordo com Allen et al. (1998), a diferença nos valores da evapotranspiração de referência nos dois métodos de estimativa é possível, porque existem diferenças entre a evaporação que ocorre no tanque e a evapotranspiração da cultura, havendo maiores resistências às perdas de vapor d'água por esta e maior exposição daquele.

$\mathrm{Na}$ Tabela 1 verifica-se, ainda, que os coeficientes determinados com base na ETo (PM) foram mais próximos daqueles apresentados por Marouelli et al. (2005) para a cultura da cebola; que o maior coeficiente de cultura foi encontrado no estádio que corresponde ao início da bulbificação até o início da maturação - estádio III e que os coeficientes de cultura determinados com base na ETo (TCA), nos estádios I e II foram inferiores aos propostos por Marouelli et al. (2005), 11,7 e 22,5\%, respectivamente, nos estádios III e IV, foram superiores 1 e 4,6\%, respectivamente. Esses resultados demonstram a importância da condução de experimentos in loco para determinação de coeficientes de cultura corroborando com a afirmação de Doorenbos \& Pruitt (1977) e Allen et al. (1998) no que diz respeito à calibração de coeficientes de cultura para condições climáticas específicas.

As condições climáticas registradas para cada estádio de desenvolvimento da cultura são apresentadas na Tabela 2, na qual se observa que, apesar de terem sido registrados, no estádio IV, menores valores da umidade relativa do ar, maior disponibilidade de energia e maior velocidade do vento, fatores que contribuem para maior transferência de vapor d'água para a atmosfera, em função do amadurecimento dos bulbos, a

Tabela 2. Média da umidade relativa do ar (UR), temperatura do ar (Tar), radiação solar global (Rg), velocidade do vento $(\mathrm{Vv})$ e total da precipitação pluviométrica (chuva) para os diferentes estádios de desenvolvimento da cebola

\begin{tabular}{cccccc}
\hline Estádios & $\begin{array}{c}\text { UR } \\
\mathbf{( \% )}\end{array}$ & $\begin{array}{c}\text { Tar } \\
\left({ }^{\mathbf{(}} \mathbf{C}\right)\end{array}$ & $\begin{array}{c}\mathbf{R g} \\
\left(\mathbf{M J ~ m}^{-2} \mathbf{d}^{-\mathbf{1}}\right)\end{array}$ & $\begin{array}{c}\mathbf{V v} \\
\left(\mathbf{k m ~ d}^{\mathbf{1}} \mathbf{)}\right.\end{array}$ & $\begin{array}{c}\text { Chuva } \\
(\mathbf{m m})\end{array}$ \\
I & 62,0 & 24,6 & 17,9 & 179,8 & 0,0 \\
II & 60,3 & 24,4 & 18,5 & 190,8 & 0,2 \\
III & 56,3 & 24,4 & 21,2 & 215,5 & 3,7 \\
IV & 51,4 & 25,0 & 24,5 & 236,4 & 0,0 \\
\hline
\end{tabular}

ETc média foi de 4,1 mm. Observa-se ainda, nesta tabela, que ocorreram pequenas precipitações nos estádios II e III. Resende et al. (2007) chamam a atenção para o fato de que chuvas em excesso, em qualquer etapa do desenvolvimento da cultura, afetam diretamente seu rendimento, pela maior ocorrência de doenças foliares e de raízes.

A Tabela 3 apresenta, para os tratamentos $\mathrm{T}_{1}, \mathrm{~T}_{2}$ e $\mathrm{T}_{3}, \mathrm{o}$ diâmetro e o peso médio de bulbos para as diferentes classes. Observa-se, nesta tabela, que para todas as classes o tratamento $\mathrm{T}_{3}$ foi o que apresentou os maiores diâmetros de bulbos, seguido do tratamento $T_{2}$, com exceção dos bulbos classificados como classe 4 cujo diâmetro médio foi menor que nos demais tratamentos. Observa-se ainda, na Tabela 3, que em relação ao peso de bulbos, o tratamento $T_{1}$ apresentou, em média, para a classe 4, o maior peso, 205,7 g e para a classe 1 (não comercial), o menor, 7,8 g; o tratamento $\mathrm{T}_{3}$ apresentou o maior peso médio de bulbos para as classes 1, 2 e 3, 8,7, 43,9 e 90,1 g, respectivamente. Araújo et al. (2004) encontraram peso médio de bulbo variando de 100 a $136 \mathrm{~g}$, valores considerados aceitáveis pelo consumidor quando se trata do cultivo orgânico. Costa et al. (2000) relatam que a preferência do consumidor nacional é por bulbos com 80 a $100 \mathrm{~g}$ e diâmetro transversal de 40 a $80 \mathrm{~mm}$. Para este estudo, nos três tratamentos, quanto ao peso médio, apenas os bulbos classificados como classe 3 atenderiam à preferência do consumidor nacional; quanto ao diâmetro, os classificados como classes 2, 3 e 4. Na seleção de bulbos, para todos os tratamentos foram encontrados bulbos de diâmetro inferior a $35 \mathrm{~mm}$, sendo considerados não comerciais.

Tabela 3. Diâmetro médio e peso médio de bulbos para as diferentes classes e tratamentos

\begin{tabular}{ccccccccc}
\hline & \multicolumn{4}{c}{ Diâmetro (mm) } & \multicolumn{5}{c}{ Peso (g) } \\
\cline { 2 - 9 } \cline { 3 - 9 } Trat. & \multicolumn{4}{c}{ Classes } \\
\cline { 2 - 9 } & $\mathbf{1}$ & $\mathbf{2}$ & $\mathbf{3}$ & $\mathbf{4}$ & $\mathbf{1}$ & $\mathbf{2}$ & $\mathbf{3}$ & $\mathbf{4}$ \\
$\mathrm{T}_{1}$ & 20,0 & 40,7 & 53,3 & 74,9 & 7,8 & 42,1 & 81,0 & 205,7 \\
$\mathrm{~T}_{2}$ & 20,9 & 41,7 & 54,4 & 73,8 & 8,0 & 40,1 & 83,6 & 194,1 \\
$\mathrm{~T}_{3}$ & 21,1 & 42,0 & 55,6 & 75,7 & 8,7 & 43,9 & 90,1 & 202,0 \\
\hline
\end{tabular}

Na Tabela 4 são apresentados a produção total, produção comercial, lâmina total de irrigação, teor de sólidos solúveis e acidez total titulável para os diferentes tratamentos. Em termos de lâmina total de irrigação, a maior lâmina aplicada foi no tratamento $\mathrm{T}_{2}, 427,1 \mathrm{~mm}$ e a menor no tratamento $\mathrm{T}_{1}, 413,3 \mathrm{~mm}$.

Tabela 4. Produção total $\left(\mathrm{P}_{\mathrm{Tot}}\right)$, produção comercial $\left(\mathrm{P}_{\mathrm{Com}}\right)$, lâmina total de irrigação $\left(\mathrm{L}_{\mathrm{Tot}}\right)$, sólidos solúveis (SS) e acidez titulável total (ATT) para os tratamentos: $\mathrm{T}_{1}$ (irrigação efetuada com base na ETc obtida nos evapotranspirômetros), $\mathrm{T}_{2}$ (irrigação tomando-se como base ETo - TCA) e $\mathrm{T}_{3}$ (irrigação feita com base na ETo - PM)

\begin{tabular}{|c|c|c|c|c|c|}
\hline \multirow{2}{*}{ Trat. } & $\mathbf{P}_{\mathrm{Tot}}$ & $P_{\text {com }}$ & \multirow{2}{*}{$\begin{array}{c}\mathrm{L}_{\mathrm{Tot}} \\
(\mathrm{mm})\end{array}$} & \multirow{2}{*}{$\begin{array}{c}\text { SS } \\
\left({ }^{\circ} \mathrm{B} \text { rix }\right)\end{array}$} & \multirow{2}{*}{$\begin{array}{l}\text { ATT } \\
\text { (\%) }\end{array}$} \\
\hline & \multicolumn{2}{|c|}{$\left(\mathrm{t} \mathrm{ha}^{-1}\right)$} & & & \\
\hline $\mathrm{T}_{1}$ & $51,5 \mathrm{a}$ & $51,3 \mathrm{a}$ & 413,3 & $10,3 \mathrm{a}$ & $0,09 \mathrm{a}$ \\
\hline $\mathrm{T}_{2}$ & $43,2 \mathrm{a}$ & $43,0 \mathrm{a}$ & 427,1 & $9,8 \mathrm{a}$ & $0,09 \mathrm{a}$ \\
\hline $\mathrm{T}_{3}$ & $51,7 \mathrm{a}$ & $51,5 \mathrm{a}$ & 419,7 & $10,4 \mathrm{a}$ & $0,10 \mathrm{a}$ \\
\hline CV\% & 18,04 & 18,19 & - & 5,22 & 10,65 \\
\hline
\end{tabular}

* Médias seguidas de mesmas letras não diferem entre si pelo teste de Tukey a 0,05 de probabilidade 
Em análise à interação entre a quantidade de água aplicada e o peso médio de bulbos comerciais constata-se que, apesar da menor lâmina do tratamento $\mathrm{T}_{1}$, o peso dos bulbos classificados como classes 2 e 4 foi, em média, 5 e $6 \%$, respectivamente, maior do que aqueles do tratamento $\mathrm{T}_{2}$; apenas para a classe 3 do tratamento $\mathrm{T}_{2}$, a maior lâmina de irrigação proporcionou maior peso de bulbo, 3,2\% maior que no tratamento $\mathrm{T}_{1}$. Kumar et al. (2007), verificaram, avaliando a resposta da cebola a diferentes níveis de irrigação, que o maior nível de irrigação influenciou, de forma positiva, a massa média de bulbos.

A produtividade total variou de 43,2 a $51,7 \mathrm{t} \mathrm{ha}^{-1}$ e a comercial de 43,0 a 51,5 t ha-1 , não diferindo estatisticamente entre si (Tabela 4). A produtividade total encontrada em todos os tratamentos foi superior àquela citada por Souza et al. (2008) para a cultivar Alfa São Francisco na região de Juazeiro, BA $\left(23,0 \mathrm{t} \mathrm{ha}^{-1}\right)$. O percentual da produtividade comercial em relação à produtividade total para os tratamentos $\mathrm{T}_{1}, \mathrm{~T}_{2} \mathrm{e} \mathrm{T}_{3}$ foi 99,$6 ; 99,5$ e 99,6\%, respectivamente, indicando baixa quantidade de bulbos não comerciais.

Observa-se ainda, na Tabela 4, que não houve diferença estatística entre os tratamentos para o teor de sólidos solúveis nem para a acidez total titulável; entretanto, o conteúdo de sólidos solúveis totais do tratamento $\mathrm{T}_{2}$ foi menor 4,9 e 5,8\%, comparado com os tratamentos $\mathrm{T}_{1}$ e $\mathrm{T}_{3}$, respectivamente. Mudanças no teor de sólidos solúveis totais com a irrigação podem ser causadas, provavelmente, pela demanda de água da cultura e pela melhor utilização de nutrientes sob disponibilidade ótima de umidade no solo (Vilas Boas et al., 2011). O teor de sólidos solúveis encontrados neste estudo corroboram com os valores apresentados por Araújo et al. (2004) para o cultivo da cebola no sistema orgânico e com os valores apresentados por Vilas-Boas et al. (2011) para duas cultivares de cebola.

Os valores de acidez total titulável (Tabela 4) para os tratamentos $\mathrm{T}_{1}$ e $\mathrm{T}_{2}$ foram iguais a $0,09 \%$ e para o tratamento $\mathrm{T}_{3}, 0,10 \%$. Araújo et al. (2004) encontraram o valor de $0,26 \%$ para a cultivar Alfa São Francisco e Grangeiro et al. (2008), 0,34\%. De acordo Grangeiro et al. (2008), a acidez elevada é considerada desejável para a industrialização das cebolas uma vez que expressa a porcentagem de ácido pirúvico e é um parâmetro utilizado para medir o grau de pungência (sabor e aroma).

\section{Conclusões}

1. Os coeficientes de cultura encontrados tomando-se como base a evapotranspiração de referência determinada pelo método do tanque Classe A, com o Kp calculado com dados climáticos obtidos in situ, foram bem próximos daqueles determinados pelo método Penman-Monteith padrão FAO.

2. O maior consumo de água da cultura ocorreu no estádio de maior desenvolvimento vegetativo e na formação de bulbos - estádio III, atingindo valores de até $6,2 \mathrm{~mm} \mathrm{~d}^{-1}$; para todo o período, a média foi de $4,0 \mathrm{~mm} \mathrm{~d}^{-1}$ e o total de $487,9 \mathrm{~mm}$.

3. O consumo de água da cultura varia não só com o seu desenvolvimento mas também com as condições climáticas locais.

4. A produtividade total superou a média nacional $(23.278 \mathrm{~kg}$ ha $^{-1}$ ) em torno de $122 \%$ para os tratamentos em que a irrigação foi efetuada tomando-se como base dados de ETc observados nos evapotranspirômetros e coeficientes de cultura $(\mathrm{Kc})$ e a ETo obtida pelo método de Penman-Monteith, e em $86 \%$ para o tratamento em que a irrigação foi feita com base na ETo obtida pelo método do tanque Classe A.

5. O manejo de irrigação, baseado no método do tanque Classe A, pode proporcionar produtividade elevada desde que sejam levadas em consideração as condições climáticas locais.

\section{Literatura Citada}

Allen, R. G.; Pereira, L. S.; Raes, D. Smith, M. Crop Evapotranspiration - Guidelines for computing crop water requirements. Rome: FAO, 1998. 301p. Irrigation and Drainage Paper 56

Araújo, J. F.; Costa, N. D.; Lima, M. A. C. de; Pedreira, C. M.; Santos, C. dos; Leite, W. M. Avaliação de genótipos de cebola em cultivo orgânico. Horticultura Brasileira, v.22, p.420-424, 2004.

Chaves, S. W. P.; Azevedo, B. M. de; Medeiros, J. F. de; Bezerra, F. M. L.; Morais, N. B. de. Evapotranspiração e coeficiente de cultivo da pimenteira em lisímetro de drenagem. Revista Ciência Agronômica, v.36, p.262-267, 2005.

Coopercitrus RevistaAgropecuária. Cebola: Terceira hortaliça mais produzida no mundo. São Paulo. Ed.303. 2012. <http://www. revistacoopercitrus.com.br/?pag $=$ materia $\&$ codigo $=6177>$ 15 Mai. 2012.

Costa, N. D.; Resende, G. M. de; Dias, R. de C. S. Avaliação de cultivares de cebola em Petrolina-PE. Horticultura Brasileira, v.18, p.57-60, 2000.

Doorenbos, J.; Pruitt, W. O. Guidelines for predicting crop water requirements. Rome: FAO. 1977. 156p. Irrigation and drainage Paper 24.

Grangeiro, L. C.; Souza, J. de O.; Aroucha, E. M. M.; Nunes, G. H. de S.; Santos, G. M. Características qualitativas de genótipos de cebola. Revista Ciência e Agrotecnologia, v.32, p.1087-1091, 2008.

IBGE - Instituto Brasileiro de Geografia e Estatística. Levantamento sistemático da produção agrícola. Rio de Janeiro: IBGE, v.25, p.1-88, 2012.

Kumar, S.; Imtiyaz, M.; Kumar, A.; Singh, R. Response of onion (Allium cepa L.) to different levels of irrigation water. Agricultural Water Management, v.89, p.161-166, 2007.

Lopes, O. D.; Kobayashi, M. K.; Oliveira, F. G.; Alvarenga, I. C. A.; Martins, E. R.; Corsato, C. E. Determinação do coeficiente de cultura $(\mathrm{Kc})$ e eficiência do uso de água do alecrim-pimenta irrigado. Revista Brasileira de Engenharia Agrícola e Ambiental, v.15, p.548-553, 2011.

Marouelli, W. A.; Costa, E. L.; Silva, H. R. Irrigação da cultura da cebola. Brasília: Embrapa Hortaliças. 2005. 17p. Circular Técnica, 37

Resende, G. M. de; Costa, N. D.; Souza, R. J. de. (ed.). Cultivo da cebola no Nordeste. Petrolina: Embrapa Semi-Árido, 2007. Sistemas de Produção, 3. <http:// sistemasdeproducao.cnptia.embrapa.br/FontesHTML/ Cebola/CultivoCebolaNordeste/clima.htm>. 15 Mai. 2012. 
Saha, U. K.; Khan, M. S. I.; Haider, J.; Saha, R. R. Yield and water use of onion under different irrigation schedules in Bangladesh. Japanese Journal of Tropical Agriculture, v.41, p.268-274, 1997.

Santa Olalla, F. M.; Valero, J. A. J.; Cortes, C. F. Growth and production of onion crop (Allium cepa $\mathrm{L}$.) under different irrigation scheduling. European Journal of Agronomy, v.3, p.85-92, 1994.

Shock, C. C.; Feibert, E. B. G.; Saunders, L. D. Irrigation criteria for drip-irrigated onions. HortScience, v.35, p.6366, 2000.

Silva, A. C. da; Lima, L. A.; Evangelista, A. W. P.; Martins, C. P. Evapotranspiração e coeficiente de cultura do cafeeiro irrigado por pivô central. Revista Brasileira de Engenharia Agrícola e Ambiental, v.15, p.1215-1221, 2011.

Snyder, R. L. Equation for evaporation pan to evapotranspiration conversions. Journal of Irrigation Drainage Engeneering of ASCE, v.118, p.977-980, 1992.
Sousa, I. F. de; Silva, V. de P. R. da; Sabino, F. G.; Netto, A. de O.; Silva, B. K. N.; Azevedo, P. V. Evapotranspiração de referência nos perímetros irrigados do estado de Sergipe. Revista Brasileira de Engenharia Agrícola e Ambiental, v.14, p.633-644, 2010.

Souza, J. O.; Grangeiro, L. C.; Santos, G. M.; Costa, N. D.; Santos, C. A. F.; Nunes, G. H. S. Avaliação de genótipos de cebola no semi-árido Nordestino. Horticultura Brasileira, v.26, p.97-101, 2008.

Vilas-Boas, R. C.; Pereira, G. M.; Souza, R. J. de; Consoni, R. Desempenho de cultivares de cebola em função do manejo da irrigação por gotejamento. Revista Brasileira de Engenharia Agrícola e Ambiental, v.15, p.117-124, 2011.

Vilas Boas, R. C.; Pereira, G. M.; Souza, R. J. de; Geisenhoff, L. O.; Lima Júnior, J. A. de. Desenvolvimento e produção de duas cultivares de cebola irrigadas por gotejamento. Revista Brasileira de Engenharia Agrícola e Ambiental, v.16, p.706-713, 2012. 